\title{
Event-triggered Cooperative Control
}

\author{
Dimos V. Dimarogonas and Karl H. Johansson
}

\begin{abstract}
Future multi-agent systems will include embedded microprocessors with limited resources to gather information and actuate the individual agent controller updates. In this paper we examine the stability of such scheme in a cooperative control problem where the actuation updates are event-driven, depending on the ratio of a certain measurement error with respect to the norm of the state. In the centralized case, we obtain a strictly positive lower bound in the inter-event times, while relevant, yet more conservative, results are obtained in the decentralized case.
\end{abstract}

\section{INTRODUCTION}

Recent technological advances on computing and communication resources have facilitated the control of large scale systems. Distributed approaches to the control of such systems are preferable to centralized ones due to their robustness to individual agent errors, scalability with respect to increased number of agents and reduced computational load. Several results concerning multi-agent cooperative control have appeared in recent literature involving agreement or consensus algorithms [11], [14], formation control [18], [1] and distributed estimation [12],[16].

An important issue that arises in the implementation of distributed algorithms is the realization of the communication and control actuation schemes. In that respect, a futuristic multi-agent system design may equip each agent with a small embedded micro-processor, who will be responsible for collecting information from neighboring nodes and actuating the control updates of the individual agent, according to some ruling. Scheduling of these actuation or execution times can be done in a time-driven or an event-driven fashion. The first case involves the traditional approach of sampling at pre-specified time instances, usually separated by a specific period. Since the microprocessors are assumed to be resource-limited, an event-triggered approach seems more favorable. In addition, a proper design can also preserve desired properties of the ideal continuous state-feedback system, such as stability and convergence. A comparison of time-driven and event-driven control for stochastic systems favoring the latter is found in [2]. Stochastic event-driven strategies have appeared in [13],[7]. In this paper, we use the deterministic event-triggered strategy introduced in [17].

Dimos Dimarogonas is with the Laboratory for Information and Decision Systems, Massachusetts Institute of Technology, Cambridge, MA, U.S.A. \{ddimar@mit.edu\}. Karl H. Johansson is with the KTH ACCESS Linnaeus Center, School of Electrical Engineering, Royal Institute of Technology (KTH), Stockholm, Sweden $\{\mathrm{kal}$ le j@ee.kth.se $\}$. This work was done within TAIS-AURES program (297316-LB704859), funded by the Swedish Governmental Agency for Innovation Systems (VINNOVA) and the Swedish Defence Materiel Administration (FMV). It was also supported by the Swedish Research Council, the Swedish Foundation for Strategic Research, and the EU FeedNetBack STREP FP7 project.
Similar results on deterministic event-triggered feedback control have appeared in [20],[19].

The approach of [17] involves triggering of the control actuation whenever a certain error becomes large enough with respect to the norm of the state. It is assumed that some kind of asymptotic stability holds for the nominal system and tools from perturbation analysis of nonlinear systems are used to analyze the convergence of the event-driven system. In particular, it is assumed that the nominal system is Inputto-State stable [15] with respect to measurement errors. We will show in the sequel that this framework is suitable for a class of cooperative control algorithms, namely those that can be reduced to a first order agreement problem [11], which has been proven to be ISS [9]. Both the cases of centralized and decentralized event-triggered control are considered. In the first case, it is assumed that there exists a global embedded microprocessor that collects information about the whole system and triggers the feedback events for each agent. We show that similarly to [17], there exists a lower bound on the inter-event times, i.e., the time between two consecutive actuation updates. In The decentralized case, each agent is equipped with its own embedded microprocessor that can gather only neighboring information. Similar yet more conservative results are obtained. In particular, we show that continuous evolution is enforced at each time instant for at least one agent and also provide a minimum lower bound for it; thus ensuring that the overall switched system does not reach an undesired accumulation point, i.e., it does not exhibit Zeno behavior [8].

The rest of the paper is organized as follows: Section II presents some necessary background and discusses the problem treated in the paper. The centralized case is discussed in Section III while Section IV presents relevant results for the decentralized case. Some examples are given in Section V while Section VI includes a summary of the results of this paper and indicates further research directions.

\section{BACKGROUND AND PROBlem STATEMENT}

In this section we first review some related results on algebraic graph theory that are used in the paper and proceed to describe the problem treated in this paper.

\section{A. Algebraic Graph Theory}

The following facts about algebraic graph theory are found in [6]. For an undirected graph $G$ with $N$ vertices the adjacency matrix $A=A(G)=\left(a_{i j}\right)$ is the $N \times N$ matrix given by $a_{i j}=1$, if $(i, j) \in E$ and $a_{i j}=0$, otherwise. If there is an edge $(i, j) \in E$, then $i, j$ are called adjacent. A path of length $r$ from a vertex $i$ to a vertex $j$ is a sequence 
of $r+1$ distinct vertices starting with $i$ and ending with $j$ such that consecutive vertices are adjacent. For $i=j$, this path is called a cycle. If there is a path between any two vertices of the graph $G$, then $G$ is called connected. A connected graph is called a tree if it contains no cycles. The degree $d_{i}$ of vertex $i$ is defined as the number of its neighboring vertices, i.e. $d_{i}=\{\# j:(i, j) \in E\}$. Let $\Delta$ be the $n \times n$ diagonal matrix of $d_{i}$ 's. Then $\Delta$ is called the degree matrix of $G$. The (combinatorial) Laplacian of $G$ is the symmetric positive semidefinite matrix $L=\Delta-A$. For a connected graph, the Laplacian has a single zero eigenvalue and the corresponding eigenvector is the vector of ones, 1 . We denote by $0=\lambda_{1}(G) \leq \lambda_{2}(G) \leq \ldots \leq \lambda_{N}(G)$ the eigenvalues of $L$. If $G$ is connected, then $\lambda_{2}(G)>0$.

\section{B. System Model}

Consider $N$ agents operating in $\mathbb{R}$. Note though that the results of the paper are extendable to arbitrary dimensions. Let $x_{i} \in \mathbb{R}$ denote the state of agent $i$. We assume first that agents' motion obeys the single integrator model:

$$
\dot{x}_{i}=u_{i}, i \in \mathcal{N}=\{1, \ldots, N\}
$$

where $u_{i}$ denotes the control input for each agent.

We assume that each agent has limited information on the other group members. In particular, each agent is assigned a subset $N_{i} \subset\{1, \ldots, N\}$ of the rest of the team, called agent $i$ 's communication set, that includes the agents with which it can communicate. The limited communication capabilities can be encoded in terms of an undirected communication graph $G=\{V, E\}$, which consists of a set of vertices $V=$ $\{1, \ldots, N\}$ indexed by the team members, and a set of edges, $E=\left\{(i, j) \in V \times V \mid i \in N_{j}\right\}$ containing pairs of vertices that represent inter-agent communication specifications.

\section{Event-triggered Cooperative Control}

A large number of multi-agent cooperative control problems can be reformulated as an agreement problem, i.e., a problem where all agents aim to achieve a common value of a certain quantity with limited information. This is for example the case of decentralized formation control [4], where it can be shown that the formation problem can be reduced to an agreement problem with a proper change of variables. We thus treat the first-order agreement problem for the system (1) in this paper.

The agreement control laws in [5], [11] were given by

$$
u_{i}=-\sum_{j \in N_{i}}\left(x_{i}-x_{j}\right)
$$

and the closed-loop equations of the nominal system (without quantization) were $\dot{x}_{i}=-\sum_{j \in N_{i}}\left(x_{i}-x_{j},\right), i \in\{1, \ldots, N\}$, so that $\dot{x}=-L x$, where $L$ is the Laplacian matrix of the communication graph. For a connected graph, all agents' states converge to a common agreement point which coincides with the average $\frac{1}{N} \sum_{i} x_{i}(0)$ of the initial states.

In this paper, we assume that each agent is equipped with one or more embedded microprocessors which are responsible for collecting information from neighboring agents and triggering the control actuation for each individual agent at discrete time instants.

As a motivating example, consider a team of $N$ vehicles/robots in a formation control scenario (which can be reformulated as an agreement problem) that can communicate and actuate their control laws at discrete instants. Between these instants their control law remains constant, i.e., this is a case of a switched system with piecewise constant control laws. This is different than the switching agreement problem treated in [14], where only the topology changes at discrete instants, but the control law has the form (2). In an eventtriggered setup, our aim is to render these discrete instants of time as rare as possible, without jeopardizing the desired system performance. This is for example the case in fleets of cooperating underwater vehicles, where the team can update its control action at discrete sampling instants.

\section{Problem Statement}

Consider the system (1). Both centralized and decentralized event-triggered cooperative control are treated in the paper. The control formulation and problem statement for each case are described in the sequel.

1) Centralized Event-triggered Cooperative Control: For each $i \in \mathcal{N}$, and $t \geq 0$, introduce a (state) measurement error $e_{i}(t)$. Denote the stack vector $e(t)=\left[e_{1}(t), \ldots, e_{N}(t)\right]^{T}$. The discrete time instants where the events are triggered are defined when a condition $f(e(t))=0$ holds. The sequence of event-triggered executions is denoted by: $t_{0}, t_{1}, \ldots$ As noted above, each $t_{i}$ is defined by $f\left(e\left(t_{i}\right)\right)=0$, for $i=0,1, \ldots$. To the sequence of events $t_{0}, t_{1}, \ldots$ corresponds a sequence of control updates $u\left(t_{0}\right), u\left(t_{1}\right), \ldots$. Between control updates the value of the input $u$ is held constant and equal to the last control update, i.e.,:

$$
u(t)=u\left(t_{i}\right), \forall t \in\left[t_{i}, t_{i+1}\right)
$$

and thus the control law is piecewise constant between the event times $t_{0}, t_{1}, \ldots$.

The centralized cooperative control problem treated in this paper can be stated as follows: "derive control laws of the form (3) and event times $t_{0}, t_{1}, \ldots$ that drive system (1) to an agreement point."

2) Decentralized Event-triggered Cooperative Control: In this case, there is a separate sequence of events $t_{0}^{k}, t_{1}^{k}, \ldots$ defined for each agent $k$ according to $f_{k}\left(e_{k}\left(t_{i}^{k}\right),\left\{e_{j}\left(t_{i}^{k}\right) \mid j \in\right.\right.$ $\left.\left.N_{k}\right\}\right)=0$, for $k \in \mathcal{N}$ and $i=0,1, \ldots$. Hence the separate condition $f_{k}\left(e_{k}(t),\left\{e_{j}(t) \mid j \in N_{k}\right\}\right)=0$ triggers the events for agent $k \in \mathcal{N}$. The decentralized control law for $k$ is updated both at its own event times $t_{0}^{k}, t_{1}^{k}, \ldots$, as well as at the last event times of its neighbors $t_{0}^{j}, t_{1}^{j}, \ldots, j \in N_{k}$. Thus it is of the form

$$
u_{k}(t)=u_{k}\left(t_{i}^{k},\left\{t_{i^{\prime}}^{j} \mid j \in N_{k}\right\}\right)
$$

where $i^{\prime} \triangleq \arg \min _{l \in \mathbb{N}: t_{i}^{k} \geq t_{l}^{j}}\left\{t_{i}^{k}-t_{l}^{j}\right\}$.

The decentralized cooperative control problem can be stated as follows: "derive control laws of the form (4), and event times $t_{0}^{k}, t_{1}^{k}, \ldots$, for each agent $k \in \mathcal{N}$ that drive system (1) to an agreement point." 


\section{Centralized Approach}

Consider the cooperative control problem of Sections IIC,D. We assume that the control law can be actuated only at discrete instances of time instead of being a continuous feedback. In contrast to traditional sampling approaches, in this paper it is assumed that the control law is actuated at instants triggered by events, and in particular, at times when the measurement error of the state variable reaches a certain threshold. In the case treated in this section, the control scheme is centralized and it is assumed that their exists a global microprocessor that collects information about the whole system and triggers the control actuation events for the whole team. This will be relaxed in the next section.

Following the notation given in the previous section, the state measurement error is defined by

$$
e(t)=x\left(t_{i}\right)-x(t), i=0,1, \ldots
$$

for $t \in\left[t_{i}, t_{i+1}\right)$. The choice of $t_{i}$ encoded by the function $f$ will be given in the sequel. The proposed control law in the centralized case has the form (3) and is defined as the event-triggered analog of the ideal control law (2):

$$
u(t)=-L x\left(t_{i}\right), t \in\left[t_{i}, t_{i+1}\right)
$$

The closed loop system is then given by

$$
\dot{x}(t)=-L x\left(t_{i}\right)=-L(x(t)+e(t))
$$

Similarly to [11], the state vector $x$ can be decomposed as

$$
x(t)=a(t) \mathbf{1}+\delta(t)
$$

where $a(t)=\frac{1}{N} \sum_{i} x_{i}(t)$ denotes the average of the agents' states and $\delta$ is called the disagreement vector in [11] and $\mathbf{1}$ is the vector of ones. We then have

$$
\begin{aligned}
\dot{a}=\frac{1}{N} \sum_{i} \dot{x}_{i}= & -\frac{1}{N} \sum_{i} \sum_{j \in N_{i}}\left(x_{i}(t)-x_{j}(t)\right) \\
& -\frac{1}{N} \sum_{i} \sum_{j \in N_{i}}\left(e_{i}(t)-e_{j}(t)\right)=0
\end{aligned}
$$

so that $a(t)=a(0)=\frac{1}{N} \sum_{i} x_{i}(0) \equiv a$, i.e., the notation $a=a(0)=a(t)$ will be used in the sequel. We now have

$$
\dot{x}=\dot{\delta}=-L(x+e)=-L(a 1+\delta+e)
$$

so that

$$
\dot{\delta}=-L(\delta+e)
$$

For an undirected graph, an important property of $\delta$ proven in [11] is $\delta^{T} L \delta \geq \lambda_{2}(G)\|\delta\|^{2}$ for all $\delta$ satisfying $x=a \mathbf{1}+\delta$.

A candidate ISS Lyapunov function for the disagreement dynamics (8) is $V=\frac{1}{2}\|\delta\|^{2}$. We have

$$
\begin{aligned}
& \dot{V}=\delta^{T} \dot{\delta}=-\delta^{T} L(\delta+e)=-\delta^{T} L \delta-\delta^{T} L e \leq \\
& \leq-\lambda_{2}(G)\|\delta\|^{2}+\|\delta\|\|L\|\|e\|
\end{aligned}
$$

Enforcing $e$ to satisfy

$$
\|e\| \leq \sigma \frac{\lambda_{2}(G)\|\delta\|}{\|L\|}
$$

with $\sigma>0$, we get

$$
\dot{V} \leq(\sigma-1) \lambda_{2}(G)\|\delta\|^{2}
$$

which is negative definite for $\sigma<1$.

Thus, the events are triggered when:

$$
f(e) \triangleq\|e\|-\sigma \frac{\lambda_{2}(G)\|\delta\|}{\|L\|}=0
$$

The event times are thus defined by $f\left(e\left(t_{i}\right)\right)=0$, for $i=0,1, \ldots$. At each $t_{i}$, the control is updated according to (6) and remains constant, i.e., $u(t)=-L x\left(t_{i}\right)$ for all $t \in$ $\left[t_{i}, t_{i+1}\right)$. Once the control task is executed the error is reset to zero, since at that point we have $e\left(t_{i}\right)=x\left(t_{i}\right)-x\left(t_{i}\right)=0$ for the specific event time so that (9) is enforced.

Similarly to [17], this control policy attains a strictly positive lower bound on the inter-event times. This is proven in the following theorem:

Theorem 1: Consider system $\dot{x}=u$ with the control law (6),(10) and assume that $G$ is connected. Then for any initial condition in $\mathbb{R}^{N}$ the inter-event times $\left\{t_{i+1}-t_{i}\right\}$ implicitly defined by the event rule (10) are lower bounded by a strictly positive time $\tau$ which is given by

$$
\tau=\frac{\sigma \lambda_{2}(G)}{\|L\|\left(\|L\|+\sigma \lambda_{2}(G)\right)}
$$

Proof: Similarly to the proof of the main result in [17], we compute the time derivative of $\frac{\|e\|}{\|\delta\|}$ :

$$
\begin{aligned}
\frac{d}{d t} \frac{\|e\|}{\|\delta\|} & =-\frac{e^{T} \dot{\delta}}{\|e\|\|\delta\|}-\frac{\delta^{T} \dot{\delta}}{\|\delta\|^{2}} \frac{\|e\|}{\|\delta\|} \\
& \leq \frac{\|e\|\|\dot{\delta}\|}{\|e\|\|\delta\|}+\frac{\|\dot{\delta}\|}{\|\delta\|} \frac{\|e\|}{\|\delta\|}=\left(1+\frac{\|e\|}{\|\delta\|}\right) \frac{\|\dot{\delta}\|}{\|\delta\|} \\
& \leq\left(1+\frac{\|e\|}{\|\delta\|}\right) \frac{\|L\|(\|\delta\|+\|e\|)}{\|\delta\|}=\|L\|\left(1+\frac{\|e\|}{\|\delta\|}\right)^{2}
\end{aligned}
$$

Using the notation $y=\frac{\|e\|}{\|\delta\|}$, we have $\dot{y} \leq\|L\|(1+y)^{2}$, so that $y$ satisfies the bound $y(t) \leq \phi\left(t, \phi_{0}\right)$, where $\phi\left(t, \phi_{0}\right)$ is the solution of

$$
\dot{\phi}=\|L\|(1+\phi)^{2}, \phi\left(0, \phi_{0}\right)=\phi_{0}
$$

Hence the inter-event times are bounded from below by the time $\tau$ that satisfies

$$
\phi(\tau, 0)=\sigma \frac{\lambda_{2}(G)}{\|L\|}
$$

We can easily see that

$$
\phi(\tau, 0)=\frac{\tau\|L\|}{1-\tau\|L\|}
$$

so that

$$
\tau=\frac{\sigma \lambda_{2}(G)}{\|L\|\left(\|L\|+\sigma \lambda_{2}(G)\right)}
$$

and the proof is complete. $\diamond$ 
Using the extension of La Salle's Invariance Principle for hybrid systems [10], the following Corollary regarding the convergence of the closed-loop system is now evident:

Corollary 2: Consider system $\dot{x}=u$ with the control law (6),(10) and assume that $G$ is connected. Then all agents converge to their initial average, i.e., $\lim _{t \rightarrow \infty} x_{i}(t)=a=$ $\frac{1}{N} \sum_{i} x_{i}(0)$ for all $i \in \mathcal{N}$.

Proof: By virtue of Theorem 1, the closed-loop switched system does not exhibit Zeno behavior. Moreover, $V(\delta)$ is positive definite and continuous and its derivative is negative definite in continuous evolution intervals. By Theorem IV.1 in [10], we have that $\lim _{t \rightarrow \infty} \delta(t)=0$, which is equivalent to $\lim _{t \rightarrow \infty} x_{i}(t)=a=\frac{1}{N} \sum_{i} x_{i}(0)$ for all $i \in \mathcal{N} . \diamond$

\section{DeCEntralized ApProach}

The approach of the previous section was centralized, in the sense that agents had to be aware of the global measurement error $e$ in order to enforce the constraint (9). In this section, we formulate a decentralized version of the problem. Each agent now updates its own control input at event times it decides based on information from its adjacent agents. The event times for each agent $i \in \mathcal{N}$ are denoted by $t_{0}^{i}, t_{1}^{i}, \ldots$. We will follow the structure described at the end of Section II to define the functions $f_{i}, i \in \mathcal{N}$ according to which the event times for agent $i$ are defined.

The measurement error for agent $i$ is defined as

$$
e_{i}(t)=x_{i}\left(t_{k}^{i}\right)-x_{i}(t), t \in\left[t_{k}^{i}, t_{k+1}^{i}\right)
$$

The control strategy for agent $i$ is now given by:

$$
u_{i}(t)=-\sum_{j \in N_{i}}\left(x_{i}\left(t_{k}^{i}\right)-x_{j}\left(t_{k^{\prime}}^{j}\right)\right)
$$

where $k^{\prime} \triangleq \arg \min _{l \in \mathbb{N}: t_{k}^{i} \geq t_{l}^{j}}\left\{t_{k}^{i}-t_{l}^{j}\right\}$. Hence, each agent takes into account the last update value of each of its neighbors in its control law. The control law for $i$ is updated both at its own event times $t_{0}^{i}, t_{1}^{i}, \ldots$, as well as at the event times of its neighbors $t_{0}^{j}, t_{1}^{j}, \ldots, j \in N_{i}$.

We then have

$$
\begin{aligned}
\dot{x}_{i}(t) & =-\sum_{j \in N_{i}}\left(x_{i}\left(t_{k}^{i}\right)-x_{j}\left(t_{k^{\prime}}^{j}\right)\right)= \\
& =-\sum_{j \in N_{i}}\left(x_{i}(t)-x_{j}(t)\right)-\sum_{j \in N_{i}}\left(e_{i}(t)-e_{j}(t)\right)
\end{aligned}
$$

Using again the decomposition $x(t)=a(t) \mathbf{1}+\delta(t)$, we have $\dot{a}=0$, so that $\dot{\delta}=\dot{x}=-L(x+e)=-L(\delta+e)$, as before. Consider $V=\frac{1}{2}\|\delta\|^{2}=\frac{1}{2} \sum_{i} \delta_{i}^{2}$. Then

$$
\dot{V}=\delta^{T} \dot{\delta}=-\delta^{T} L(\delta+e)=-\delta^{T} L \delta-\delta^{T} L e
$$

so that

$$
\begin{aligned}
\dot{V} \leq & -\lambda_{2}(G)\|\delta\|^{2}-\delta^{T} L e= \\
& -\lambda_{2}(G) \sum_{i} \delta_{i}^{2}-\sum_{i} \sum_{j \in N_{i}} \delta_{i}\left(e_{i}-e_{j}\right)
\end{aligned}
$$

and thus,

$$
\begin{aligned}
\dot{V} & \leq-\lambda_{2}(G) \sum_{i} \delta_{i}^{2}+\sum_{i} \sum_{j \in N_{i}}\left|\delta_{i}\right|\left|e_{i}-e_{j}\right| \\
& \leq-\lambda_{2}(G) \sum_{i}\left(\delta_{i}^{2}-\left|\frac{\delta_{i}}{\lambda_{2}(G)}\right| \sum_{j \in N_{i}}\left(\left|e_{i}\right|+\left|e_{j}\right|\right)\right)
\end{aligned}
$$

Enforcing the condition

$$
\sum_{j \in N_{i}}\left(\left|e_{i}\right|+\left|e_{j}\right|\right) \leq \lambda_{2}(G) \sigma_{i}\left|\delta_{i}\right|
$$

we get

$$
\sigma_{i} \delta_{i}^{2} \geq\left|\frac{\delta_{i}}{\lambda_{2}(G)}\right| \sum_{j \in N_{i}}\left(\left|e_{i}\right|+\left|e_{j}\right|\right)
$$

so that

$$
\dot{V} \leq-\lambda_{2}(G) \sum_{i}\left(\delta_{i}^{2}-\sigma_{i} \delta_{i}^{2}\right)=-\lambda_{2}(G) \sum_{i}\left(1-\sigma_{i}\right) \delta_{i}^{2}
$$

which is negative semidefinite for $0<\sigma_{i}<1$.

Thus for each $i$, an event is triggered when

$$
f_{i}\left(e_{i},\left\{e_{j} \mid j \in N_{i}\right\}\right)=0
$$

where $f_{i}\left(e_{i},\left\{e_{j} \mid j \in N_{i}\right\}\right) \triangleq \sum_{j \in N_{i}}\left(\left|e_{i}(t)\right|+\left|e_{j}(t)\right|\right)-$ $\lambda_{2}(G) \sigma_{i}\left|\delta_{i}(t)\right|$. The update rule (14) holds at the event times $t_{k}^{i}$ corresponding to agent $i$ : $f_{i}\left(e_{i}\left(t_{k}^{i}\right),\left\{e_{j}\left(t_{k}^{i}\right) \mid j \in N_{i}\right\}\right)=0$ with $k=0,1, \ldots$ and $i \in \mathcal{N}$. At an event time $t_{k}^{i}$, we have $e_{i}\left(t_{k}^{i}\right)=x_{i}\left(t_{k}^{i}\right)-$ $x_{i}\left(t_{k}^{i}\right)=0$, and since $\sum_{j \in N_{i}}\left(\left|e_{i}(t)\right|+\left|e_{j}(t)\right|\right) \geq \sum_{j \in N_{i}}\left|e_{j}(t)\right|$ for all $t \geq 0$, the condition (13) is enforced.

Remark: Although (14) is verified by agent $i$ only based its own and neighboring agents' information, it does require some global information, in the sense that agents need to know the values of $\lambda_{2}(G)$ and $a$ in order to check (14). We note that this condition has been relaxed in our later work [3], using a different event-triggered formulation. In particular, in the condition derived in [3], each agent only needs to know the sum of relative states and the number of its neighbors to implement it. Hence knowledge of the aforementioned global parameters is relaxed.

The following theorem regarding the inter-event times holds in the decentralized case:

Theorem 3: Consider system $\dot{x}_{i}=u_{i}, i \in \mathcal{N}=$ $\{1, \ldots, N\}$ with the control (12) and update rule (14), and assume that $G$ is connected. Then for any initial condition and any time $t \geq 0$ there exists at least one agent $k \in \mathcal{N}$ for which the next inter-event interval is strictly positive.

Proof: We assume that (14) holds for all $i \in \mathcal{N}$ at time $t$. If it doesn't hold, then continuous evolution is possible since at least one agent can still let its absolute measurement error increase without resetting (11). Hence assume that at $t$ all errors are reset to zero. We will show that there exists at least one $k \in \mathcal{N}$ such that its next inter-event interval is bounded from below by a certain time $\tau_{D}>0$. 
First note that the term $\sum_{j \in N_{i}}\left(e_{i}(t)+e_{j}(t)\right)$ is the $i$-th row of the vector $(\Delta+A) e$, where $\Delta$ is the degree matrix of $G$ and $A$ its adjacency matrix. We then have

$$
\frac{\sum_{j \in N_{i}}\left(\left|e_{i}(t)\right|+\left|e_{j}(t)\right|\right)}{\|\delta\|} \leq \frac{\|\Delta+A\|\|e\|}{\|\delta\|}
$$

for each $i \in \mathcal{N}$. Denoting $k=\arg \max _{i}\left|\delta_{i}\right|$ the maximum element of $\|\delta\|$, we have

$$
\frac{\sum_{j \in N_{k}}\left(\left|e_{k}(t)\right|+\left|e_{j}(t)\right|\right)}{N\left|\delta_{k}\right|} \leq \frac{\|\Delta+A\|\|e\|}{\|\delta\|}
$$

so that

$$
\frac{\sum_{j \in N_{k}}\left(\left|e_{k}(t)\right|+\left|e_{j}(t)\right|\right)}{\left|\delta_{k}\right|} \leq N \frac{\|\Delta+A\|\|e\|}{\|\delta\|}
$$

Using now the proof of Theorem 1 and (14), the next interevent interval of agent $k$ is bounded from below by a time $\tau_{D}$ that satisfies

$$
N\|\Delta+A\| \frac{\tau_{D}\|L\|}{1-\tau_{D}\|L\|}=\sigma_{k} \lambda_{2}(G)
$$

so that

$$
\tau_{D}=\frac{\sigma_{k} \lambda_{2}(G)}{\|L\|\left(N\|\Delta+A\|+\sigma_{k} \lambda_{2}(G)\right)}
$$

and the proof is complete. $\diamond$

Please note that the result of this Theorem is more conservative than the centralized case, since it only guarantees that there are no accumulation points and continuous evolution is viable at all times instants. However, no lower bound similar to the one of Theorem 1 is provided. In that sense, the result on the decentralized case is rather preliminary and more work will be devoted to providing a strictly positive inter-event time of the overall switched system in the future.

On the other hand, the result of Theorem 3 guarantees that the overall switched system does not exhibit Zeno behavior, i.e., there are no infinite switches in finite time. Using now La Salle's Invariance Principle for Hybrid Systems [10], the following convergence result is straightforward:

Corollary 4: Consider system $\dot{x}=u$ with the control law (12),(14) and assume that $G$ is connected. Then all agents converge to their initial average, i.e., $\lim _{t \rightarrow \infty} x_{i}(t)=a=$ $\frac{1}{N} \sum x_{i}(0)$ for all $i \in \mathcal{N}$.

Proof: By virtue of Theorem 3, the closed-loop switched system does not exhibit Zeno behavior. The rest of the proof is identical to that of Corollary 2. $\diamond$

\section{EXAMPLES}

In this section we provide some computer simulated examples to support our results. We consider a network of four agents whose neighboring sets are given by $N_{1}=$ $\{2,3\}, N_{2}=\{1,3\}, N_{3}=\{1,2,4\}, N_{4}=\{3\}$. We consider both the centralized and the decentralized framework. Four agents start from random initial conditions and evolve under the control (6),(10) in the first case, and (12),(14) in the second case. We have $\lambda_{2}(G)=1$ and $\|L\|=4$ in this example, and have also set $\sigma=0.65$ for the centralized, and $\sigma_{1}=\sigma_{2}=0.55$ and $\sigma_{3}=\sigma_{4}=0.65$ for the decentralized control example. Figure 1 shows the evolution of the norm of $\|x(t)-a \mathbf{1}\|$ in both cases in time. The top solid line shows the evolution of $\|x(t)-a \mathbf{1}\|$ in the centralized and the bottom dotted line in the decentralized case. It can be seen that the system reaches the agents' initial average as $t \rightarrow \infty$ in both frameworks.

Figure 2 shows the evolution of the error norm in the centralized case. The solid line represents the evolution of $\|e(t)\|$. This stays below the specified state-dependent threshold $\|e\|_{\max }=\sigma \frac{\lambda_{2}(G)\|\delta\|}{\|L\|}$ which is represented by the dotted line in the Figure. The existence of a minimum inter-event time is clearly visible. We have $\tau=0.05$ in this example, which is an overestimation of the simulated bound, which in this example can be computed to be $0.2 \mathrm{~ms}$.

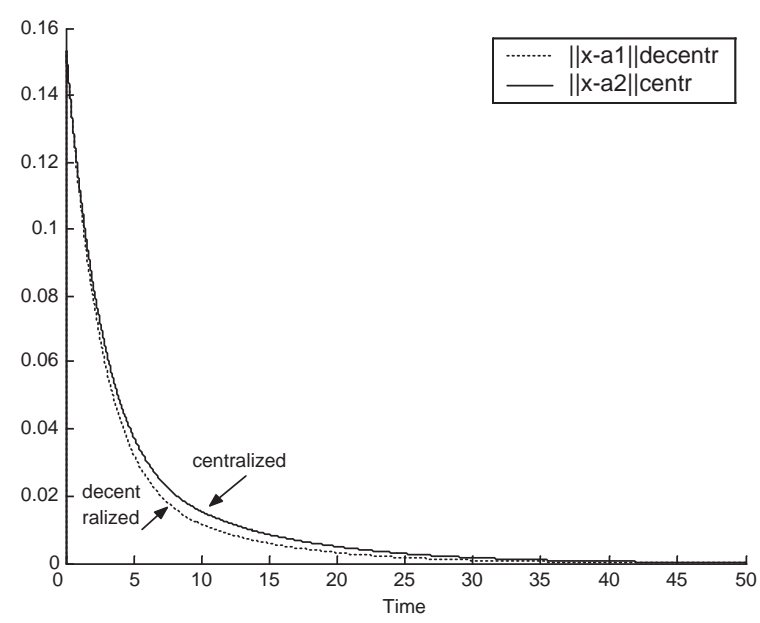

Fig. 1. Four agents evolve under (6),(10) in the centralized case, and the control law (12),(14) in the decentralized case. We have $\lambda_{2}(G)=1,\|L\|=$ 4 and $\sigma=0.65, \sigma_{1}=\sigma_{2}=0.55$ and $\sigma_{3}=\sigma_{4}=0.65$. Convergence to the initial average is achieved in both cases.

The next two figures depict how condition (13) is realized in the decentralized case for agents 2,4. The solid line in Figure 3 shows the evolution of the sum $\left|e_{2}(t)\right|+\left|e_{1}(t)\right|+\left|e_{3}(t)\right|$. This stays below the specified state-dependent threshold given by (13) $M_{2}=\lambda_{2}(G) \sigma_{2}\left|\delta_{2}\right|$ which is represented by the dotted line in the Figure. The same holds for agent 4 as shown in Figure 4 where the solid line represents the sum $\left|e_{3}(t)\right|+\left|e_{4}(t)\right|$ which also stays below the specified state-dependent threshold given by (13) $M_{4}=\lambda_{2}(G) \sigma_{4}\left|\delta_{4}\right|$, represented by the dotted line in the Figure.

\section{SUMMARY AND FUTURE WORK}

Cooperative control schemes of multi-agent systems under event-triggered actuation update rules were proposed and analyzed. We examined the stability of such schemes considering actuation updates are event-driven, depending on the ratio of a certain measurement error with respect to the 


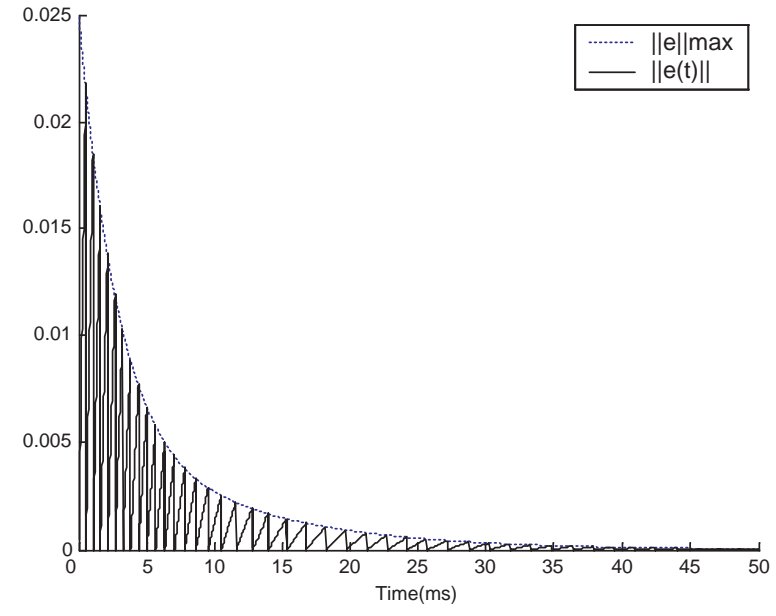

Fig. 2. Evolution of the error norm in the centralized case. The solid line represents the evolution of the error norm $\|e(t)\|$, which stays below the specified state-dependent threshold $\|e\|_{\max }=\sigma \frac{\lambda_{2}(G)\|\delta\|}{\|L\|}$ which is represented by the dotted line in the Figure.

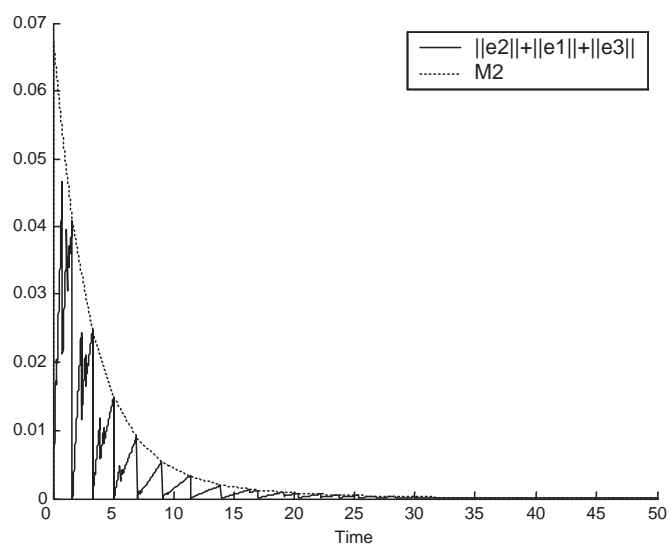

Fig. 3. Four agents are controlled by (12),(14) in the decentralized case Condition (13) is depicted in the this case for agent 2 . The solid line shows the evolution of the sum $\left|e_{2}(t)\right|+\left|e_{1}(t)\right|+\left|e_{3}(t)\right|$. This stays below the specified state-dependent threshold given by (13) $M_{2}=\lambda_{2}(G) \sigma_{2}\left|\delta_{2}\right|$ which is represented by the dotted line.

norm of the state. In the centralized case, we obtained a strictly positive lower bound in the inter-event times, while relevant, yet more conservative, results were obtained in the decentralized case. Future research will focus on providing rules that guarantee better bounds on the inter-event time intervals in the decentralized case.

\section{REFERENCES}

[1] M. Arcak. Passivity as a design tool for group coordination. IEEE Transactions on Automatic Control, 52(8):1380-1390, 2007.

[2] K.J. Astrom and B. Bernhardsson. Comparison of Riemann and Lebesgue sampling for first order stochastic systems. 41st IEEE Conference on Decision and Control, pages 2011-2016, 2002.

[3] D.V. Dimarogonas and K.H. Johansson. Event triggered control for multi-agent systems. 48th IEEE Conference on Decision and Control, 2009. submitted

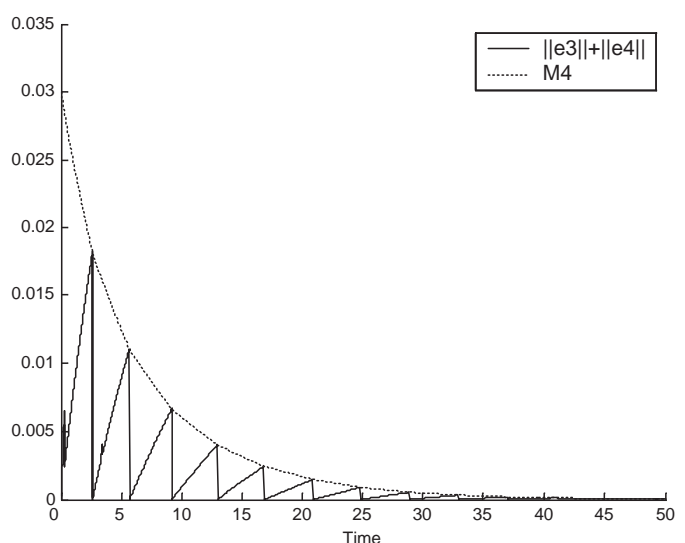

Fig. 4. Condition (13) is depicted in the this case for agent 4. The solid line shows the evolution of the sum $\left|e_{4}(t)\right|+\left|e_{3}(t)\right|$. This stays below the specified state-dependent threshold given by (13) $M_{4}=\lambda_{2}(G) \sigma_{4}\left|\delta_{4}\right|$ which is represented by the dotted line.

[4] D.V. Dimarogonas and K.J. Kyriakopoulos. A connection between formation infeasibility and velocity alignment in kinematic multi-agent systems. Automatica, 44(10):2648-2654, 2008.

[5] J.A. Fax and R.M. Murray. Graph Laplacians and stabilization of vehicle formations. 15th IFAC World Congress, 2002.

[6] C. Godsil and G. Royle. Algebraic Graph Theory. Springer Graduate Texts in Mathematics \# 207, 2001.

[7] E. Johannesson, T. Henningsson, and A. Cervin. Sporadic control of first-order linear stochastic systems. Hybrid Systems: Computation and Control, pages 301-314, 2007.

[8] K.H. Johansson, M. Egerstedt, J. Lygeros, and S.S. Sastry. On the regularization of zeno hybrid automata. Systems and Control Letters, 38:141-150, 1999.

[9] D.B. Kingston, W. Ren, and R. Beard. Consensus algorithms are inputto-state stable. American Control Conference, pages 1686-1690, 2005.

[10] J. Lygeros, K.H. Johansson, S. Simic, J. Zhang, and S. Sastry. Dynamical properties of hybrid automata. IEEE Transactions on Automatic Control, 48(1):2-17, 2003.

[11] R. Olfati-Saber and R.M. Murray. Consensus problems in networks of agents with switching topology and time-delays. IEEE Transactions on Automatic Control, 49(9):1520-1533, 2004.

[12] R. Olfati-Saber and J.S. Shamma. Consensus filters for sensor networks and distributed sensor fusion. 44th IEEE Conference on Decision and Control, pages 6698-6703, 2005.

[13] M. Rabi, K.H. Johansson, and M. Johansson. Optimal stopping for event-triggered sensing and actuation. 47th IEEE Conference on Decision and Control, 2008.

[14] W. Ren and R. Beard. Consensus seeking in multiagent systems under dynamically changing interaction topologies. IEEE Transactions on Automatic Control, 50(5):655-661, 2005.

[15] E.D. Sontag. On the input-to-state stability property. European Journal of Control, 1:24-36, 1995.

[16] A. Speranzon, C. Fischione, and K.H. Johansson. Distributed and collaborative estimation over wireless sensor networks. 45th IEEE Conference on Decision and Control, pages 1025-1030, 2006.

[17] P. Tabuada. Event-triggered real-time scheduling of stabilizing control tasks. IEEE Transactions on Automatic Control, 52(9):1680-1685, 2007.

[18] H.G. Tanner, A. Jadbabaie, and G.J. Pappas. Flocking in fixed and switching networks. IEEE Transactions on Automatic Control, 52(5):863-868, 2007.

[19] X. Wang and M.D. Lemmon. Event-triggered broadcasting across distributed networked control systems. American Control Conference, 2008.

[20] X. Wang and M.D. Lemmon. Self-triggered feedback control systems with finite-gain L2 stability. IEEE Transactions on Automatic Control, 54(3):452-467, 2009. 